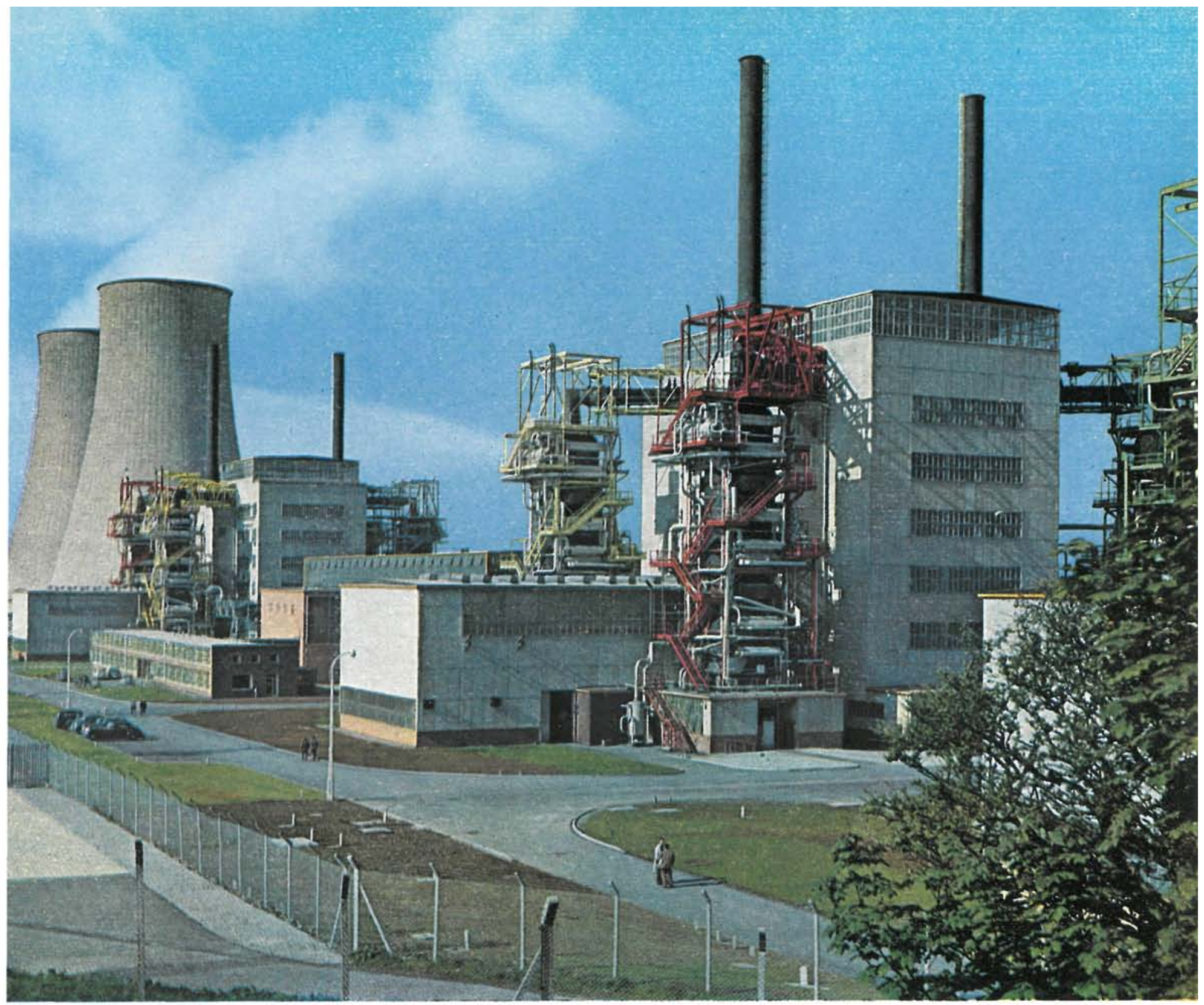

\title{
calder hall
}

\section{central para el aprovechamiento de energía nuclear}

Información facilitada por la United Kingdom Atomic Energy Authority

La nueva central nuclear de Calder Hall, situada en el noroeste de Inglaterra, próxima a las costas de Cumberland, ha constituido el primer paso decisivo que plasma en realidad lo que hasta hace poco no salia de la via de ensayos y laboratorios: es decir: el aprovechamiento de la energia nuclear en los medios pacíficos industriales. A los ingleses corresponde, pues, el primer puesto en esta vanguardia inquieta del progreso científico del que tanto se espera.

Aunque la central se halla próxima al mar, no implica esto la necesidad de grandes muelles de atraque para su aprovisionamiento de materias primas, carbón u otro tipo cualquiera de los combustibles ordinarios, cuyo consumo es voluminoso en las centrales termoeléctricas importantes. Una carretera relativamente modesta servirá para el abastecimiento de la central.

Contiguo a la central de Calder Hall se halla la de Windscale. Se dedica ésta a la preparación de plutonio, elemento utilizable en el proceso nuclear para la obtención de calor en su fisión. 
La central de Calder Hall, cuya capacidad total se eleva a 4 grupos de turbo-alternadores de $23.000 \mathrm{~kW}$ de potencia cada uno, entró en servicio, suministrando energía a la red nacional inglesa el 17 de octubre de 1956, a cuya inauguración oficial asistió la reina.

\section{Ligera idea del proceso nuclear en la central}

Una central térmica ordinaria en la que se genera energia eléctrica se compone, esencialmente, de cuatro partes principales: una fuente generadora de calor (combustible), la transformación de agua en vapor (caldera), utilización de la energía en la expansión del vapor (turbina) $y$ la transformación de la energía dinámica en eléctrica (alternador). De estos cuatro agentes, sólo el primero se diferencia en los dos tipos de aprovechamientos. En las térmicas ordinarias el calor se btiene por la combinación quimica ordinaria del combustible con el oxígeno, reacción $u$ oxidación en la que los átomos se combinan entre ellos sin perder su integridad, mientras que en el proceso nuclear el átomo se altera en su propio núcleo produciendo calor, fenómeno que se ha denominado fisión.

En la naturaleza sólo existe una clase de átomo que permite este tipo de alteración: el uranio. Su peso en la escala atómica es 235. Al obtener el uranio de las minas, se distinguen dos tipos siderenciales, químicamente iguales, pe ro su peso es diferente. De 140 partes, una es uranio U 235 cuya fisión es capaz de producir calor. Y el resto, ligeramente más pesado, se ha denominado U 238 .

Para provocar la fisión del U 235 es necesario el choque con un neutrón, partícula que poseen todos los núcleos ató micos de los distintos elementos, a excepción del hídrógeno. Al aparecer la fisión los núcleos se subdividen en dos partes aproximadamente iguales, llamadas productos de la fisión, dando lugar a la generación de calor y desprendiendo dos o tres neutrones que en a gran velocidad. Este hecho es de la mayor importancia, ya que estos neutrones pueden chocar con otros átomos U 235 $\mathrm{y}$ continuar la fisión ininterrumpidamente, dando por resultado una producción regular continuada de un flujo de lar contion.
calor.
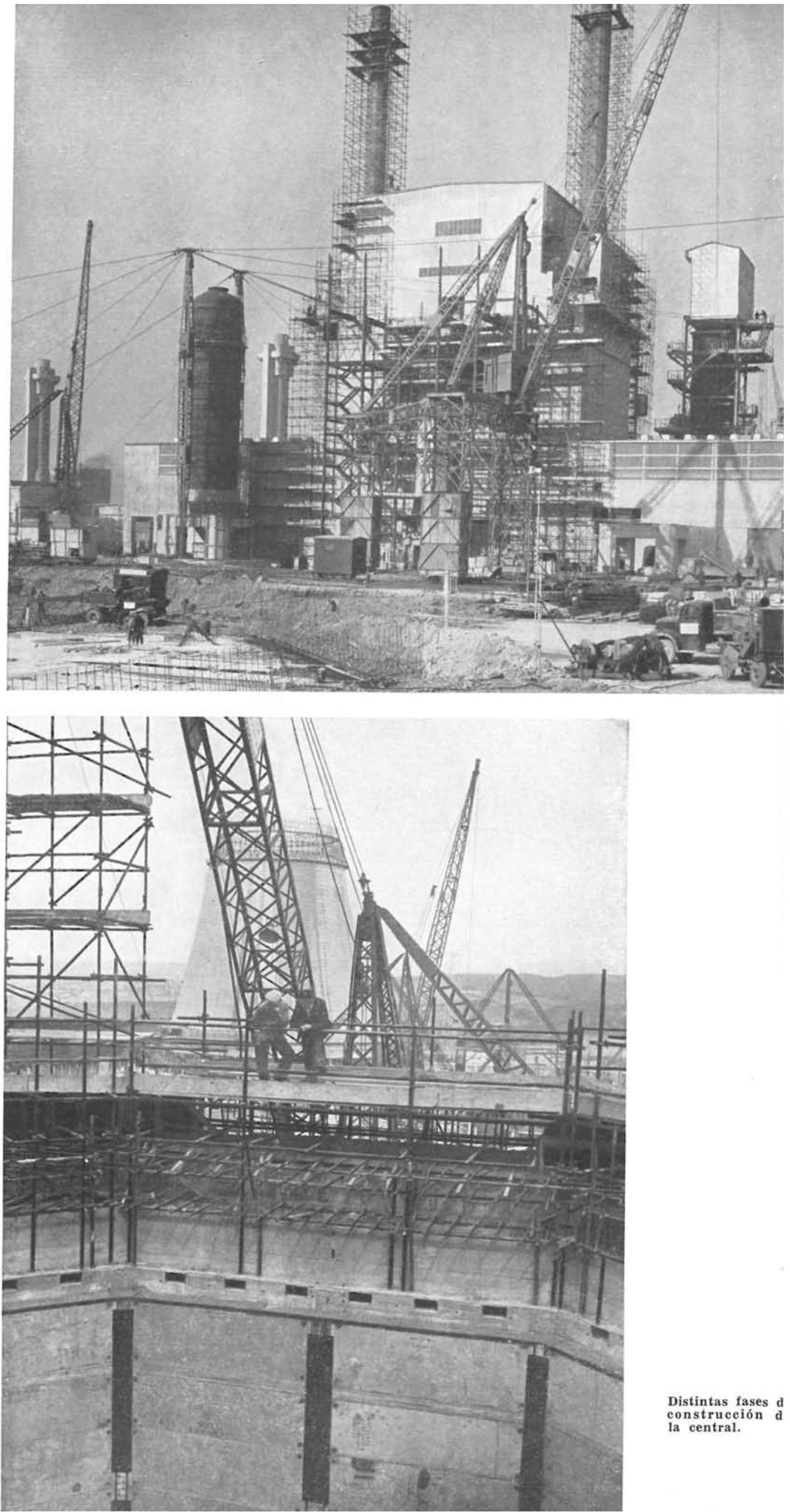

Distintas fases d construcción d la central. 


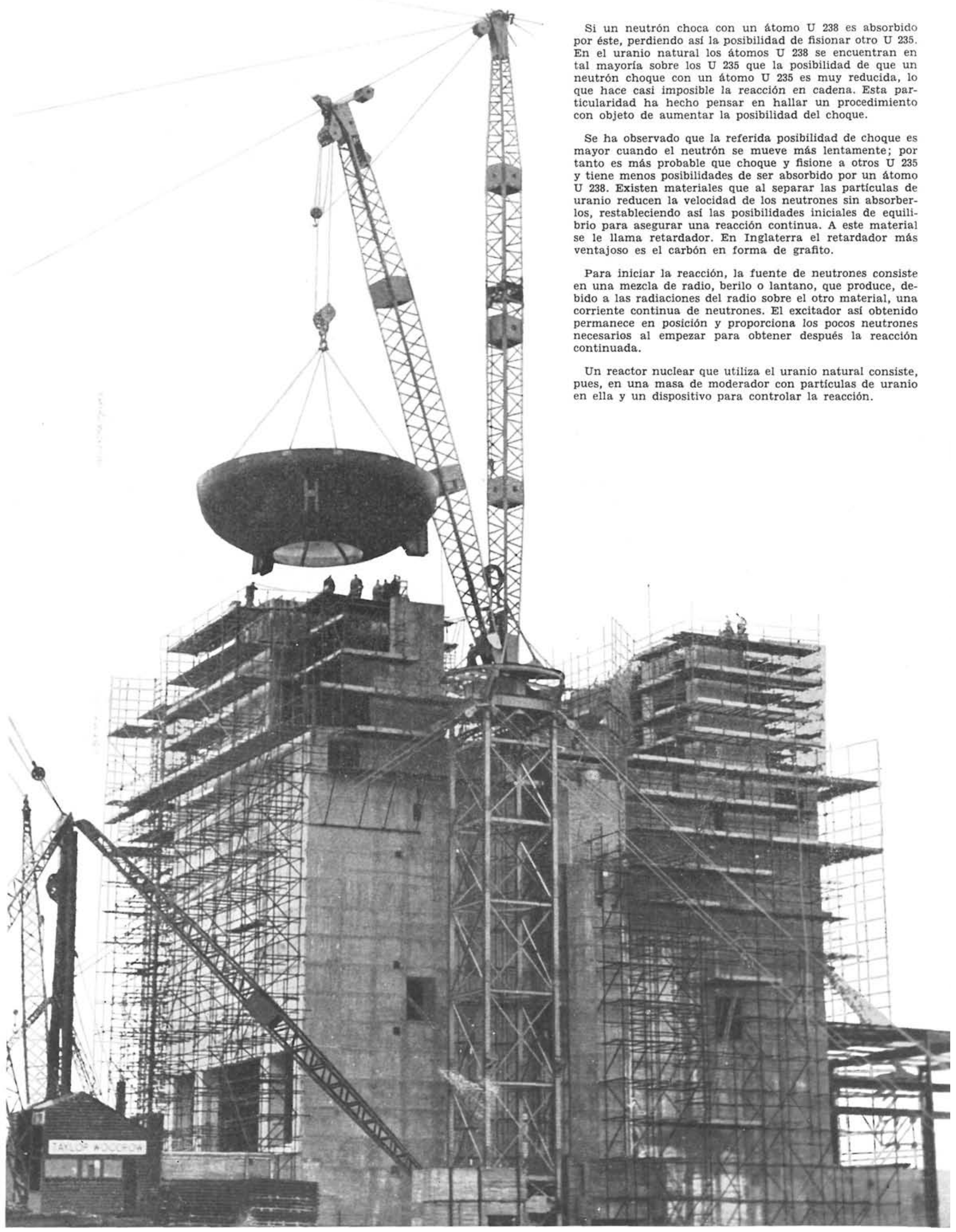



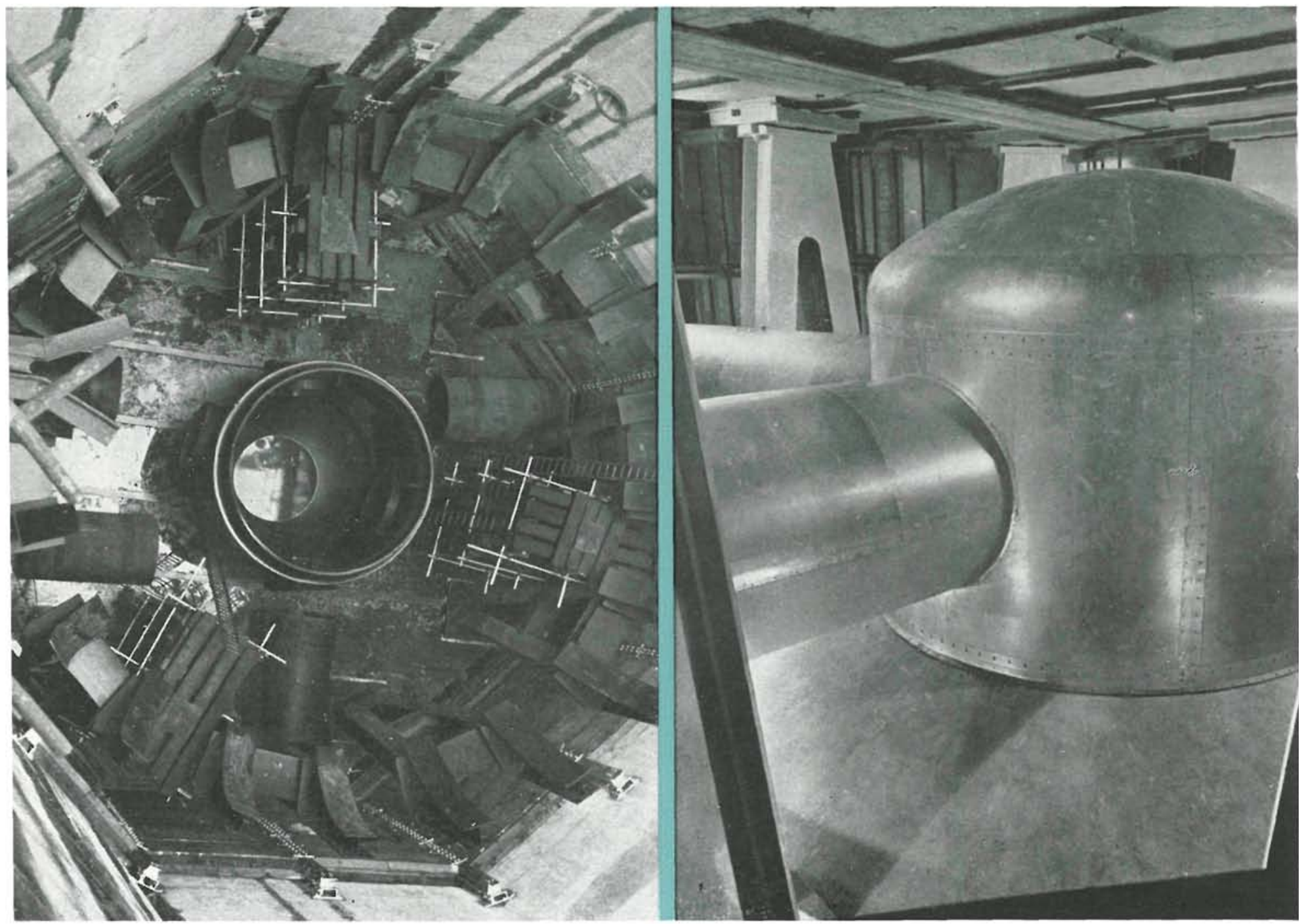

Interior y exterior de la toma de CO.

Este dispositivo regulador está constituído por una serie de varillas que contienen boro, elemento capaz de absorber neutrones, y que, metiendo las más o menos en el reactor, se logra ajustar el número de neutrones a las condiciones necesarias para lograr la fisión y generación de calor regularmente.

A parte de la gran cantidad de calor generada en el interior del reactor, que debe salir ayudándose de un medio apropiado, ya en estado líquido o gaseoso, las radiaciones de la fisión son nocivas biológicamente al organismo humano, por lo que hay que protegerse contra ellas con una pantalla o escudo.

Los neutrones, al chocar con el U 238 son absorbidos por éste sin producir calor; pero esto no supone una pérdida real, aun cuando no ayuden a mantener la continuidad del proceso de fisión. Los átomos U 238 que absorben un neutrón experimentan una reacción diferente que los convierte, con el tiempo, en un nuevo elemento artificial llamado plutonio, que, como el U 235 , es capaz de fisionarse y producir calor. Como el U 235, es un combustible de alta concentración para el reactor.

Para darnos una idea de la potencia calorifica de estos combustibles observaremos que la fisión de 450 gr de U 235 equivale, en calor, al que daría la combustión de 1.000 toneladas de carbón. Parece, pues, que con una pequeña cantidad de uranio la central podría funcionar durante un tiempo considerable; pero no sucede aś, ya que el reactor requiere un núcleo de ciertas dimensiones llamadas criticas, porque, de lo contrario, muchos de los neutrones escapan de su superficie y no se puede lograr la fisión en cadena; y, si se emplea uranio natural, que es un "combustible» muy diluído, el tamaño del núcleo es de alrededor de un metro $y$, por tanto, se necesitan toneladas de uranio para que se verifique la fisión continuada. 

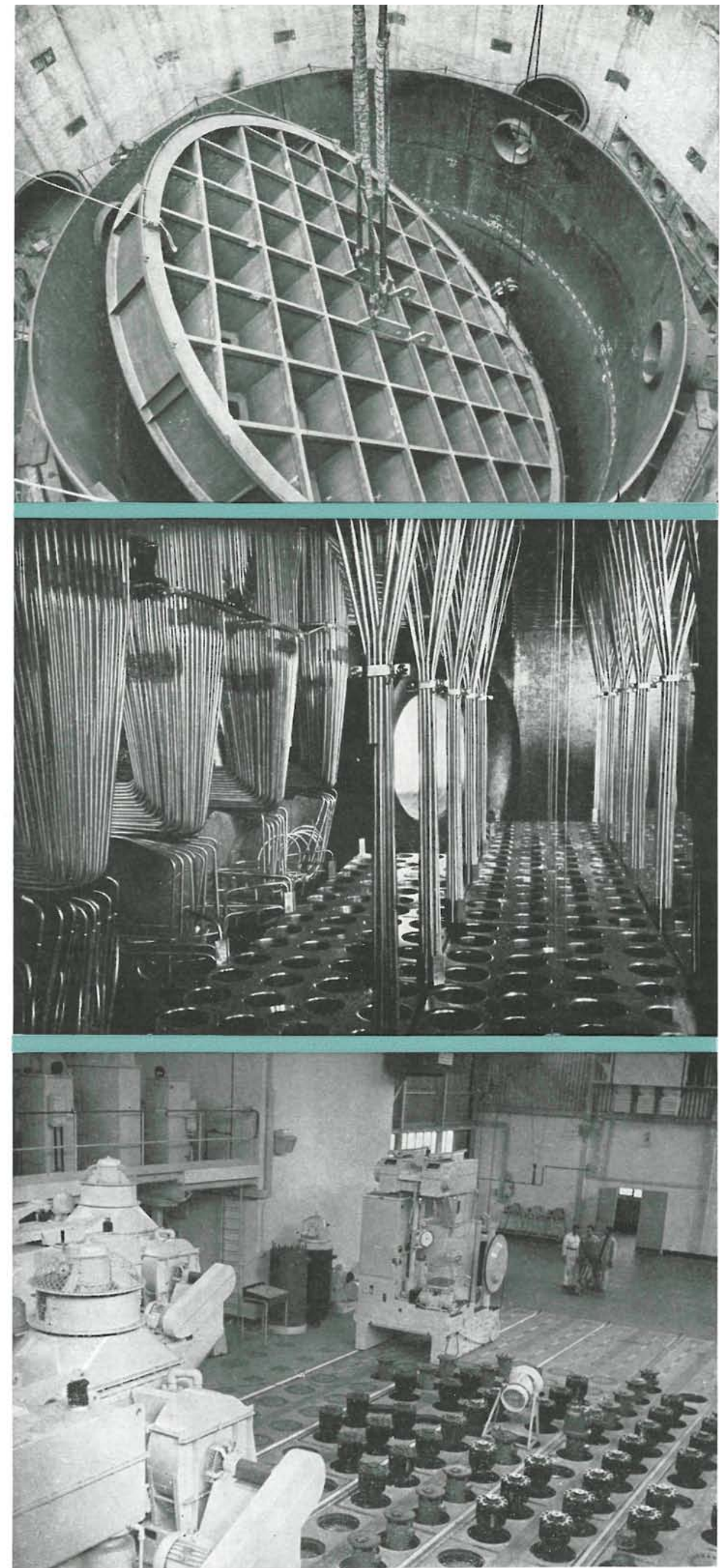

Soporte del reactor.

Tubos detectores de fugas $y$ estructura de grafito.
Planta de carga del reactor. 


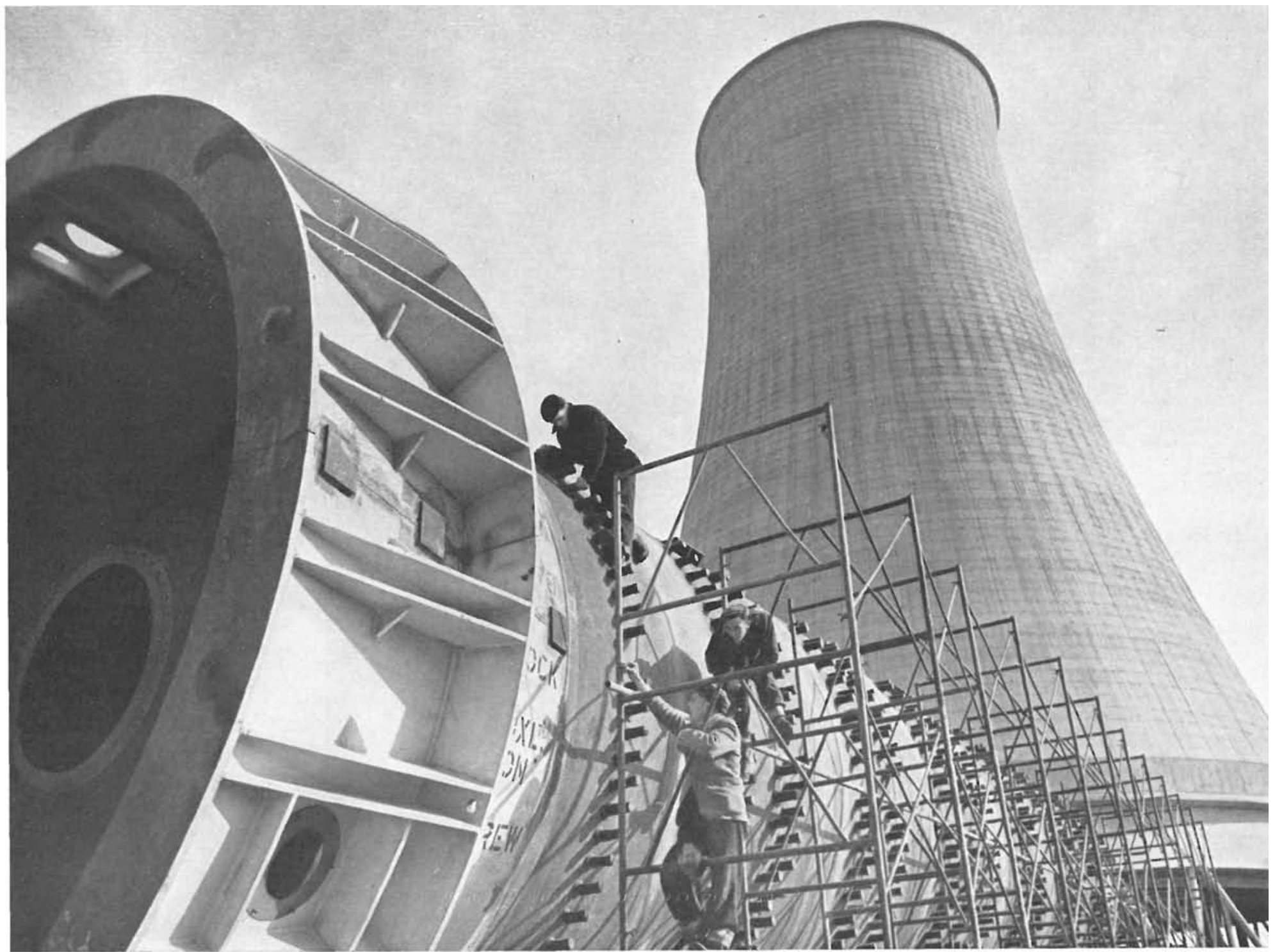

Montando el dispositivo de intercambio de calor.

Además, los núcleos de "combustible» uranio, suministrados en forma de varillas, se han de sellar en recipientes antes de introducirlos en el reactor, para que no se escapen los productos de la fisión, que son altamente radiactivos, así como para evitar la oxidación del uranio. Al uranio en barras se le llama "combustible».

Las llamadas "cenizas» de la combustión nuclear permanecerán en el reactor y absorben neutrones; por tanto, cuanto más tiempo están en el reactor las varillas, mayor es la pérdida de U 235 , y la absorción de neutrones va aumentando hasta hacer la fisión imposible. En este momento se sustituyen las varillas usadas, llevándolas a la factoría para tratarlas, recuperar el U 235 y plutonio y dejarlas nuevamente en estado para su nuevo reempleo.

El reactor de Calder Hall, de $11 \mathrm{~m}$ de anchura y 8,2 de altura, consiste en una masa de 58.000 ladrillos de varias formas, a través de los cuales se han dejado 1.800 huecos o taladros, de los que 1.696 se reservan a las varillas de «combustible»-compuestas de 6 elementos de $1 \mathrm{~m}$ de longitud cada una-, colocados unos encima de otros. Los restantes agujeros se han previsto para las varillas de control. Estas varillas de uranio son de unos $25 \mathrm{~mm}$ de diámetro, y se hallan en el interior de recipientes de magnesio convenientemente sellados. El exterior de estas varillas tiene una serie de espirales formando aletas para aumentar la superficie de disipación del calor.

Para sacar el calor del interior del reactor, éste se ha envuelto de una pared metálica, de $50 \mathrm{~mm}$ de espesor, que constituye la cámara de presión, de $11,30 \mathrm{~m}$ de dímetro y $21,30 \mathrm{~m}$ de altura, en cuyo interior se hace pasar una corriente de bióxido de carbono a $7 \mathrm{~kg} / \mathrm{cm}^{2}$ de presión, para lo cual se dispone de cuatro salidas en la parte superior y otras cuatro en la inferior.

En la parte superior de la campana de presión se hallan: el dispositivo para la carga de varillas «combustibles» y las de control de marcha; estas yarillas pasan a través del escudo biológico de hormigón. Para evitar el hacer muchos agujeros en la campana de presión, por cada agujero pasan 16 varillas.

Puede suceder que uno de los recipientes o envolturas de las varillas se agrieta y dé lugar a fugas, y con ellas a la salida de materias radiactivas; en cuyo caso, y con ayuda de un detector, se localiza la varilla y se sustituye por otra.

Los gases salientes del reactor se llevan a la parte superior de las torres de recuperación de calor, donde una serie de tubos, llenos de agua en la parte inferior, y vapor en la superior, lo aprovechan para suministrar vapor a los turbo-generadores. 

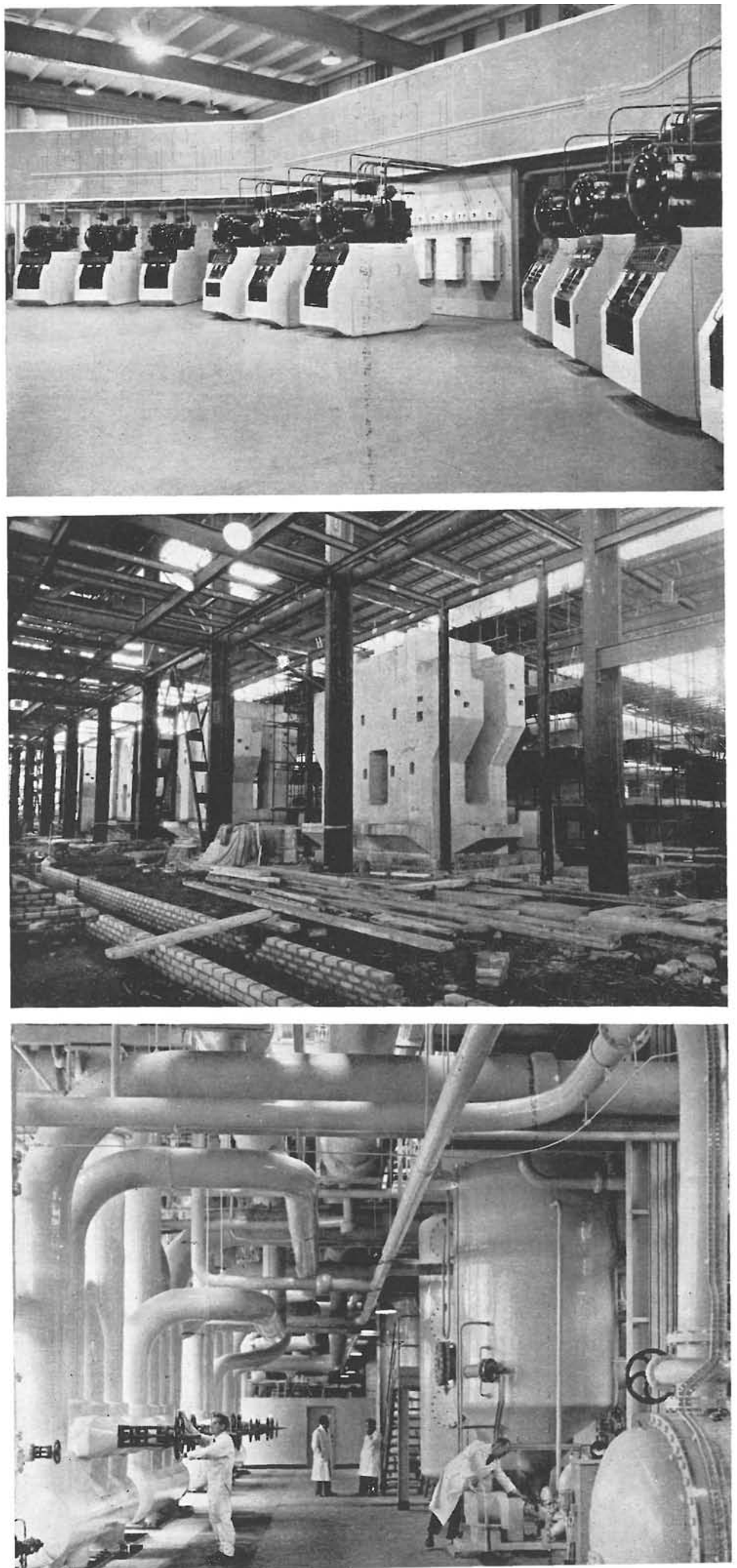

Detectores de fugas de escorias. Sala de turbinas.

Estas torres metálicas, convenientemente aisladas, tienen 24,4 metros de altura y 5,20 de diámetro.

La separación entre estas torres y la sala de turbo-generadores aisla a ésta de toda posible contaminación radiactiva.

E1 equipo generador eléctrico está compuesto de cuatro grupos, de $23.000 \mathrm{~kW}$ de potencia cada uno. La instalación de esta maquinaria es análoga a otra de tipo convencional, con la unica variante pecial que permite, emergencia en los grupos turboalternadores, condensar el vapor generado en el reactor. Complementan las instalaciones dos torres para la refrigeración del agua de los cc sadores, puesa no se dispone de agua de refrigeración en suficiente cantidad para permitir una sola pasada de ésta.

\section{Méfodos construcfivos}

La construcción de esta central ha planteado serios problemas de orden técnico y plazo para su terminación. La falta material de tiempo exigió se construyeran los muros de hormigón que rodean al reactor sin esperar al montaje de la campana metálica, que va en su interior y sirve de cámara de presión. Al terminar en altura estos muros o escudo biológico, se procedió a colocar, por piezas de enorme peso, preparadas previamente en taller, las seis partes que se acoplarian y soldarian en el interior del escudo. Esta operación se realizó ayudándose de un puente provisional tipo Bailey y de potentes grúas.

La losa de hormigón armado que cierra el escudo es de $2,40 \mathrm{~m}$ de espesor; debajo de ella va el escudo térmico, formado de chapa de $15 \mathrm{~cm}$ de espesor. Entre 18 losa y este escudo metálico se formó una cámara de aire o refrigeración, a la que no debian caer grumos durante el hormigonado de la losa de cierre.

Como base de apoyo de cada uno de los reactores se construyó una solera de hormigón, de $39 \times 31,4 \mathrm{~m}$ en planta y 3,4 de espesor. Sobre esta losa se 1evantó el escudo biológico, que consiste en un prisma octogonal hueco, de hormigón, de $14 \mathrm{~m}$ entre caras interiores y $2,15 \mathrm{~m}$ de espesor. En esta construcción, de $27,40 \mathrm{~m}$ de altura, se exigió gran precisión para conservar la verticalidad de paramentos y 2,5 de densidad en el hormigón. Esta densidad tiene gran importancia si se tiene en cuenta la función de estas paredes: aislar y ser impermeables a todas las radiacio nes. 
Una vez realizados los muros laterales del reactor, se procedió a montar y soldar las distintas partes de la campana de presión o recinto envolvente: se empezó por la base, donde entran los tubos que inyectan el bióxido de carbono; se siguió con una especie de reticulado plano intermedio, que sirve de apoyo a las barras de uranio y masa de grafito, y se terminó en la parte superior, de donde arrancan los tubos de toma de gases calientes.

Para liberar de tensiones las chapas de la campana después de soldarla, se preparó una resistencia eléctrica que se extiende sobre toda la superficie interna de la misma. Utilizando una potencia de $1.500 \mathrm{~kW}$ se logró aumentar la temperatura del metal hasta $500^{\circ} \mathrm{C}$, bajando después gradualmente dicha temperatura hasta la normal. A continuación se probó la cámara sometiéndola a una presión de $9,50 \mathrm{~kg} / \mathrm{cm}^{2}$, se limpió escrupulosamente su interior y se montó la masa de grafito, observando un meticuloso cuidado en la limpieza y aseo del personal, enseres y materiales que en el interior del reactor se empleaban hasta dejarlo completamente terminado.

Para hormigonar y colocar el escudo térmico de chapa de $15 \mathrm{~cm}$ de espesor debajo de la losa de hormigón armado de $2,40 \mathrm{~m}$ de espesor, se tuvieron que vencer algunas dificultades, pues entre el intradós de la losa y el escudo térmico queda una altura de sólo $15 \mathrm{~cm}$. Esto obligó a suspender el fondo metálico del encofrado perdido de unos puentes provisionales tipo Bailey. Al fondo de! encofrado se le dió una contraflecha apropiada para contrarrestar la flecha que la gran carga del hormigón pudiera ocasionar en los puentes.

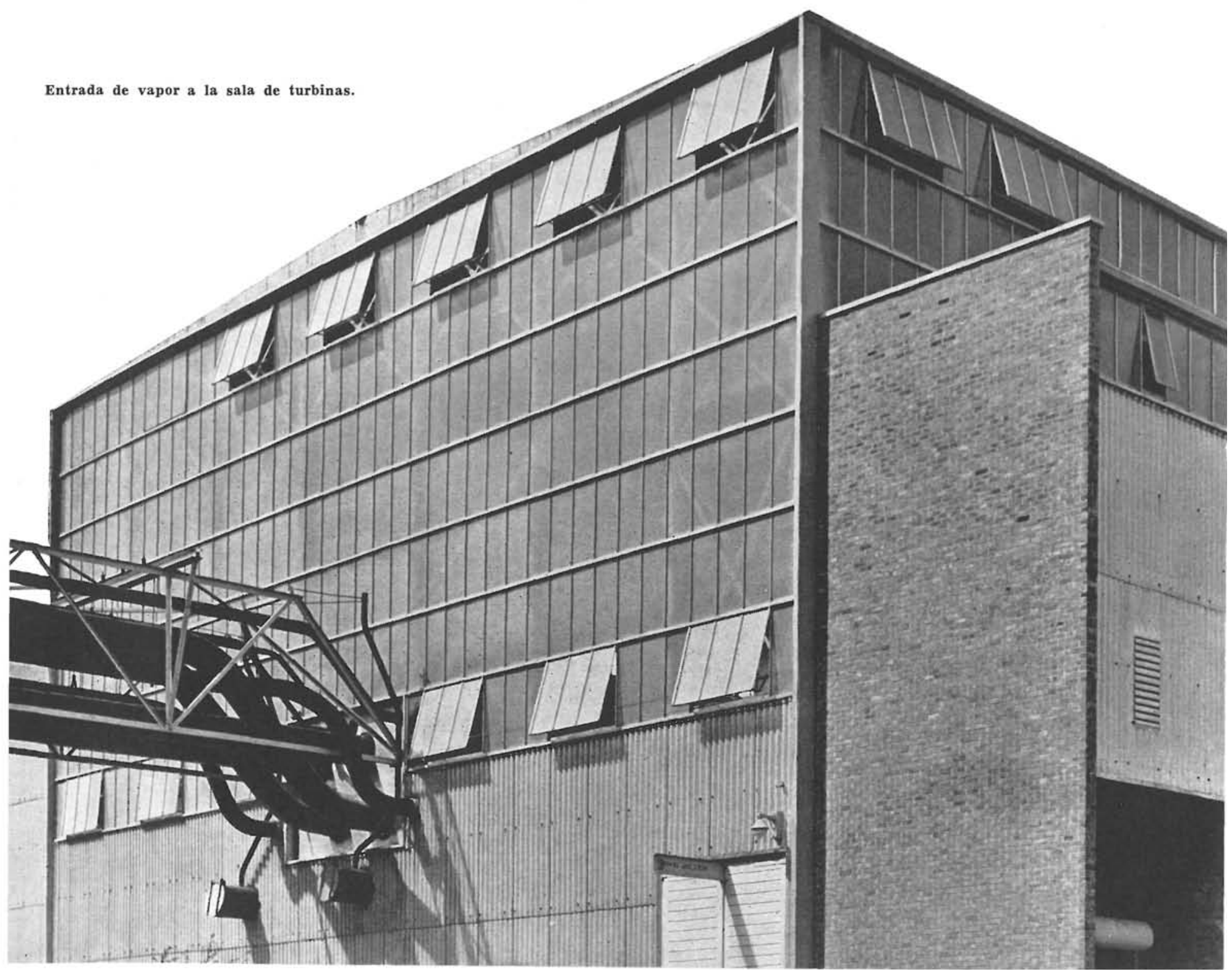




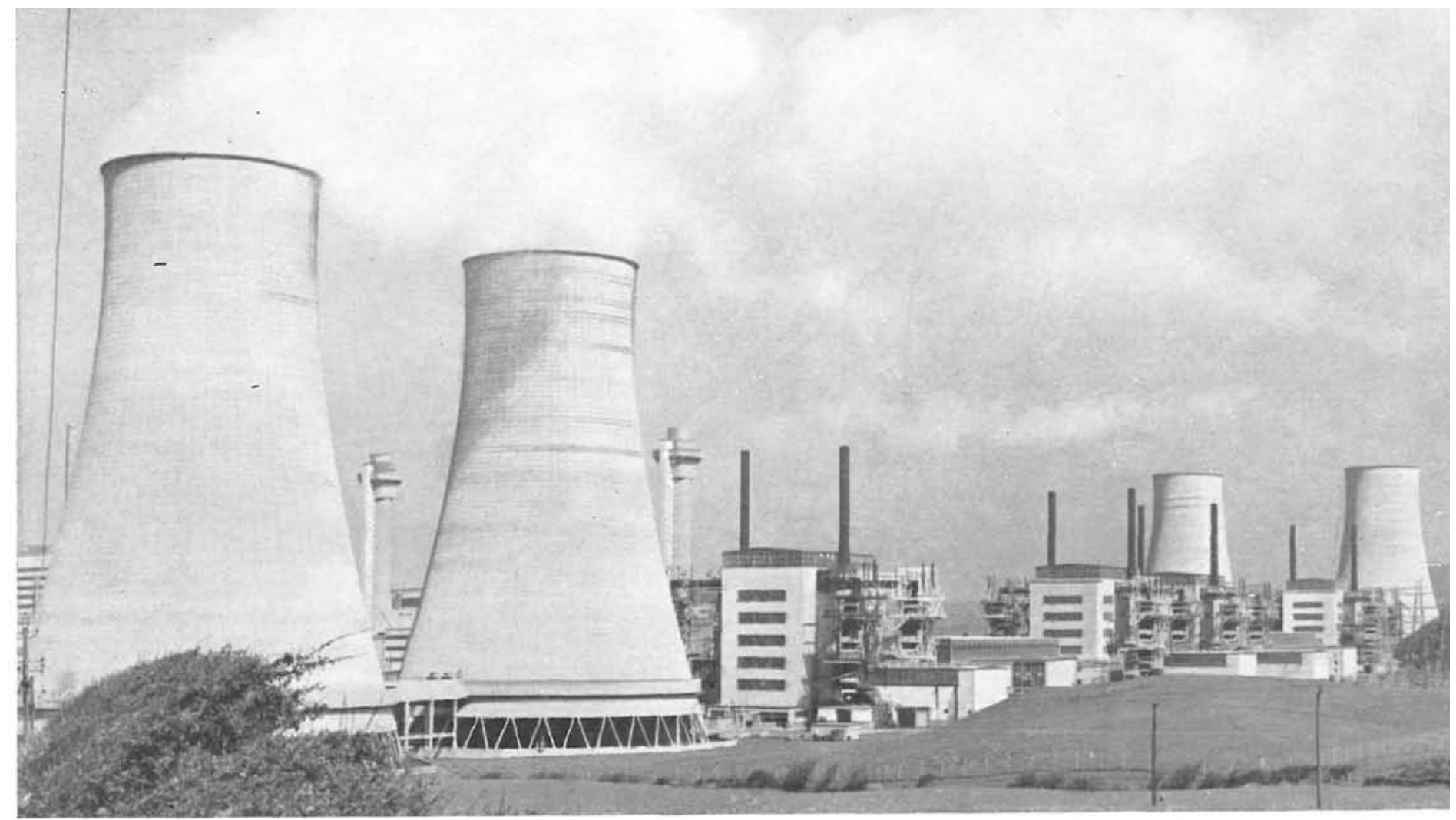

Dado el gran espesor de la losa, ésta se hormigonó formando cuatro capas sucesivas. Después de fraguar el hormigón se cortaron los tirantes de suspensión, se retiraron los puentes y quedó terminada la losa. Todas estas operaciones se ejecutaron dentro de las dificultades propias de las armaduras, tubos y falta de espacio.

Los grandes recipientes metálicos para la recuperación del calor, cuatro por reactor, en cuyo interior se halla una gran cantidad de tubos, que desempeñan la función de caldera generadora de vapor, se apoyan, cada uno de ellos, sobre una solera de hormigón. Su construcción y montaje en obra ha sido muy similar a los procedimientos empleados en las envolventes metálicas campanas de los reactores.

Adosados a los reactores se hallan las salas de los ventiladores, que impelen el bióxido de carbono utilizado en la instalación como vehículo de transporte del calor generado en el reactor.

La sala de turbinas, torres de refrigeración, subestación eléctrica y oficinas, se han construído siguiendo los métodos tradicionales.

Vista general.

Soportes perimetrales de una torre de refrigeración.

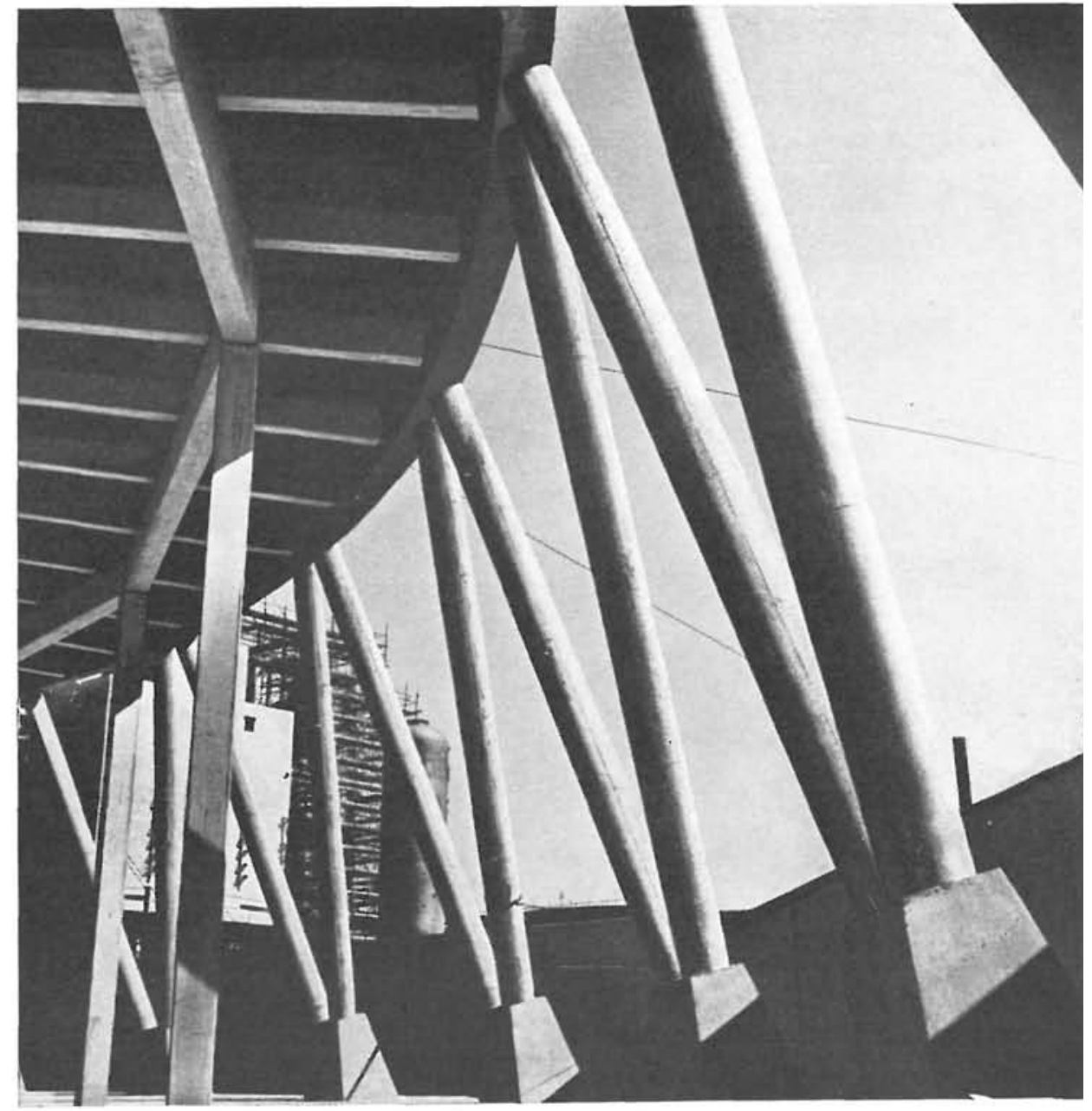

